\title{
Copper nanoparticles Synthesis supported in diatoms
}

\author{
Cristian Miranda1, Felipe Sanhueza ${ }^{1,2}$ \\ ${ }^{1}$ Techmological Development Unit (UDT), University of Concepción, Chile \\ c.miranda@udt.cl \\ ${ }^{2}$ Department of Materials Engineering, University of Concepción, Chile
}

\section{Extended Abstract}

Within the antimicrobial agents, copper has been demostrate have very good bactericidal properties and until now there is no record of copper-resistant bacteria [1]. In 2008, the Environmental Protection Agency (EPA) of the United States approving copper and the registration of more than 270 their metal alloys which prevented the growth of bacteria and other microorganisms, such as fungi and viruses [2].

On the other hand, the diatomous earth or diatomite are a biogenic mineral resource in abundance. It has an antifungal and antibacterial effect by contact, used in organic crops [3]. In addition, they are chemically inert and have a large surface area of mass, the diatoms are excellent carriers and dispersants of chemical products. In water, diatomite is able to absorb up to 2.5 times its weight. [4].

For this reason, that the development of composite copper nanostructures supported in diatoms as antibacterial agents is a great opportunity for to increase the antibacterial performance mixing both material obtaining a sinergic effect, especially a part of an antimicrobial by nature, where studies that the bactericidal activity is related to one side with the antibacterial effect of The metal ions released from the nanoparticle surfaces and the antibacterial effect of the diatoms themselves for use as support.

Copper nanoparticles are synthesized in the presence of diatoms by polyol method, from $0.2 \mathrm{M}$ copper sulfate solution, the reduction of copper was obtain using sodium borohidride as a reductor (method A) and citric acid (method B) $[5,6]$. The synthesis of copper nanoparticles was carried out in basic medium, to guarantee the stability of the reducing agent. According to Liu et al. (2012) [6] Copper at basic $\mathrm{pH}$ can be reduced by three routes, as well as the formation of nanoparticles.

From the SEM images, the typical structure of the naked diatoms can be observed prior to the synthesis process (Figure 1a) and the copper nanoparticles supported in the diatoms (Figure 2b). It is possible to observe the typical dendritic structure of the copper nanoparticles, which are recovering the diatoms, some diatoms without copper are also identified.
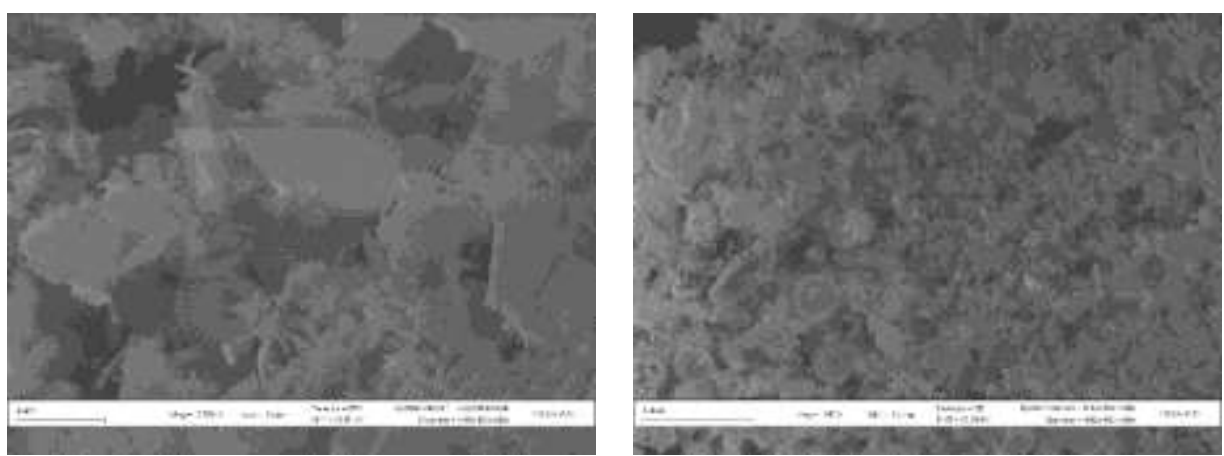

Fig. 1: SEM images diatoms (a); copper nanoparticles supported in diatoms (b).

Figure 2 shows the antibacterial effect of copper nanoparticles supported in diatoms synthesized by both methods, compared with the antibacterial capacity of copper nanoparticles and diatoms on Escherichia Coli ATCC 25922. 


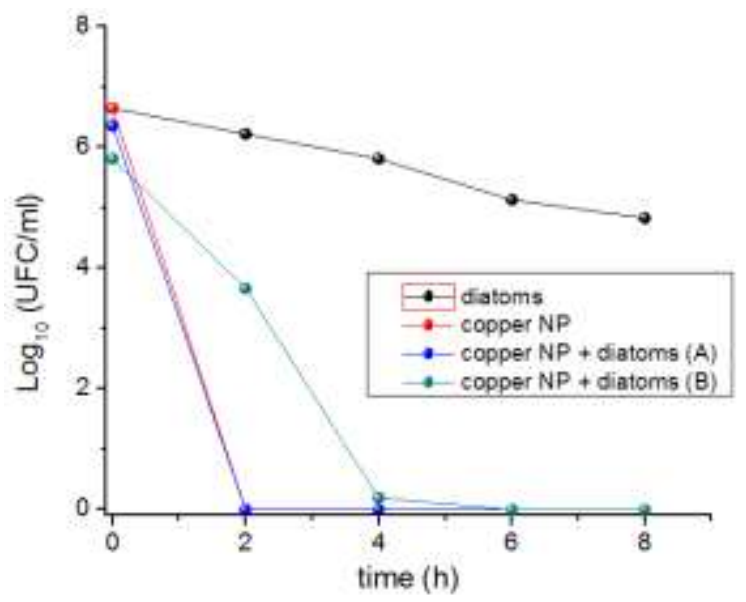

Fig. 2: Antibacterial performance.

The graph shows the rapid decrease of the bacterial population by contact of copper nanoparticles supported in diatoms, reaching a percentage of survival of $0 \%$ after $1 \mathrm{~h}$. Copper nanoparticles reach the total bacterial death at $4 \mathrm{~h}$ contact, while the diatoms show a low efficiency. This shows that the antibacterial activity is greater in both synthesis methods, compared to the copper nanoparticles and diatoms separately, which demonstrates the synergistic effect of the combination of both materials.

\section{References}

[1] C. Espírito Santo, E. W. Lam, C. G. Elowsky, D. Quaranta, D. W. Domaille, C. J. Chang, G. Grass, "Bacterial killing by dry metallic copper surfaces," Appl. Environ. Microbiol., vol. 77, no. 3, pp. 794-802, 2011.

[2] "Guidelines for Environmental Infection Control in Health-Care Facilities Report," Infection, Control \& Prevention, USEPA, 2008.

[3] A. C. Viso, D. Pesando, C. Baby, "Antibacterial and Antifungal properties of some marine diatoms in culture," Botanica Marina, vol. 30, pp. 41-45, 2009.

[4] Robert Digman, Kenneth Lohman, "Late Pleistocene Diatoms from the Arica Area, Chile; Art. No 78," U.S. Geol. Survey, Prof. Paper, pp. C69-72, 2014.

[5] A. M Beaird, T.A. Davis, and M.A. Matthews, "Deliquescence in the Hydrolysis of Sodium Borohydride by Water Vapor," Industrial \& Engineering Chemistry Research, vol. 49, no. 20, pp. 9596-9599, 2010.

[6] Q. M. Liu, et al., "Preparation of $\mathrm{Cu}$ nanoparticles with $\mathrm{NaBH} 4$ by aqueous reduction method," Transactions of Nonferrous Metals Society of China, vol. 22, no. 1, pp. 117-123, 2012. 